Kwong, V.H.S., Fang, Z., Gibbons, T.T., Parkinson, W.H. \& Smith, P.L., 1993, ApJ, 411, 431 Nussbaumer, H., 1977, A\&A, 58, 291

Shull, J.M., Snow, T.P. \& York, D.G., 1981, ApJ, 246, 549

Spitzer, L. \& Fitzpatrick, E.L., 1993, ApJ, 409, 299

\title{
MOLECULAR DATA FOR STELLAR OPACITIES
}

\section{UFFE GRÅE JØRGENSEN}

\section{Niels Bohr Institute, Blegdamsvej 17, DK-2100 Copenhagen, Denmark}

In total, 40 neutral diatomic molecules, 2 molecular ions, and 7 polyatomic molecules are known from observed photospheric stellar spectra. Line data for opacity computations (i.e., lists of line frequencies, intensities, and excitation energies) exist for 17 of these molecules, although the data are complete only for a handful of them. A detailed description of stellar photospheric molecules can be found in Tsuji (1986), and the existing opacity data have been reviewed by Jorgensen (1995).

Listed line frequencies in the data bases are either the measured values, or based on computed molecular constants obtained from fits to measured values. Attempts to compute $a b$ initio line frequencies have so far resulted in lower accuracy than what is obtained by use of molecular constants. Published line strengths include measured values as well as ab initio values. For strong bands the ab initio intensities are as accurate as the laboratory values, whereas measured values for weak bands are generally more accurate than the ab initio values. The primary advantage of ab initio computations is therefore that the complete set of all transitions can be obtained. Exploratory studies have shown that completeness of the line data is crucial for the obtained stellar photospheric structure.

As an alternative to the ab initio computations of the line intensities, fits to experimental data have been attempted. The most promising method seems to be to fit the dipole function by use of a Padé approximant. Combined with a potential fitted to experimental energy levels, such a dipole function can in principle be used to predict the complete list of band intensities and line intensities for all bands with energies up to the molecular dissociation energy. The part of the dipole function which corresponds to the largest stretching (or bending) of the molecule is the most uncertain in such fits as well as in ab initio computations. This part is responsible for most of the many weak transitions, and large uncertainties are therefore to be excepted in the computed intensities of the weak spectral bands. As these are of major importance for the stellar photospheric structure (due to their huge number and their pseudo continuous appearance in the spectrum), a particularly large effort is desirable in comparing computed intensities with laboratory data for a representative sample of weak bands. Unfortunately, only few measurements of weak bands exist. Attempts to measure all the existing bands are not recommended, because of 
their huge number and due to the difference in the population of states in the laboratory and in the (relatively hot) photosphere. For example, recent calculations revealed that it is necessary to account for about 85,000 vibrational transitions in the water molecule in its electronic ground state (corresponding to a few times $10^{7}$ vibrational rotational transitions) in order to obtain a good description of the water opacity in cool giant stars. publications:

Data from the following data bases are available from the authors of the following

The HITRAN data base (Rothman et al. 1992) lists frequencies, excitation energies, and strengths for 648,000 spectral lines from 31 different molecules of astrophysical and geophysical interest. The GISA data base (Husson et al. 1991) follows the HITRAN data base, but includes additional lines from molecules of particular interest for studies of the giant planets. The ATMOS data base is constructed from the two former catalogues, but includes, in addition, data for trace molecules in the Earth's atmosphere (Brown et al. 1987).

The Berkeley data base (Davis 1987, 1994) lists measured line frequencies of 6 molecules (TiO, $\mathrm{ZrO}, \mathrm{YO}, \mathrm{CN}, \mathrm{FeH}$, and $\mathrm{LaO}$ ) of particular interest for stellar astronomy. The Berkeley Newsletter is a bi-monthly literature survey concerning small molecules and ions of astrophysical interest. The RADEN data base (Kuznetsova et al. 1993) stores information on more than 2600 publications about specific molecules and transitions for 300 diatomic molecules.

Kurucz's molecular line list, or the Smithsonian data base (Kurucz \& Peytremann 1975, Kurucz 1994), contains frequencies, intensities, and excitation energies for 11 million lines from 15 diatomic molecules ( $\mathrm{TiO}, \mathrm{C}_{2}, \mathrm{SiO}, \mathrm{CN}, \mathrm{CO}, \mathrm{H}_{2}, \mathrm{CH}, \mathrm{NH}, \mathrm{OH}, \mathrm{MgH}$, and $\mathrm{SiH}$ ). The line frequencies are computed from molecular constants, and measured frequencies are listed where known. The line strengths are computed from a Rydberg-Klein-Rees potential fitted to measure energy levels, combined with a semi-empirical dipole function extrapolated from measured values. Also, the line strengths are substituted with measured values whenever available.

The SCAN data base (Jorgensen \& Larsson 1990, Jorgensen \& Jensen 1993, Jorgensen $1995)$ contains frequencies, intensities, and excitation energies for 70 million lines from 3 diatomic and 4 small polyatomic molecules ( $\mathrm{TiO}, \mathrm{CN}, \mathrm{CH}, \mathrm{HCN}, \mathrm{C}_{2} \mathrm{H}_{2}, \mathrm{C}_{3}$, and $\mathrm{H}_{2} \mathrm{O}$ ). The line frequencies are computed from molecular constants. For the computation of the line strengths, ab initio dipole functions (computed using the CASSCF method) are used together with either a Klein-Dunham potential function, a MORBID potential, or a polynomial fit to an ab initio potential.

Littleton \& Davis (1985) used the measured data from part of the Berkeley program described above to construct a list of frequencies, intensities, and excitation energies for 330,000 lines from $\mathrm{ZrO}$ and 1,200 lines from YO. 
Chackerian, Goorvich, Tipping and Pineiro fitted the potential functions and the dipole functions to experimental values for the $\mathrm{CO}, \mathrm{CS}$, and $\mathrm{SiS}$ (and SiO) molecules. The extrapolation of the dipole functions to larger stretchings than those included in the measurement, were performed by use of a Padé approximant. The resultant calculations provided data for 35,000 lines of CO (Goorvich \& Chackerian 1994), 35,000 lines of CS (Pineiro et al. 1987a), and 600 lines of SiS (Pineiro et al. 1987b).

Langhoff \& Bauschlicher (1993) listed frequencies, intensities, and excitation energies for a large number of lines of SiO. The potential function and the dipole function is based on extensive ab initio computations using the CASSCF method.

Besides the $b-b$ transitions collected in the line list data bases, also $b$ - $f$ transitions and collision induced transitions are expected to be of importance for the spectra and/or the structure of cool stars. van Dishoeck (1994) has recently reviewed the situation concerning molecular $b$ - $f$ transitions, and Borysow (1994) has reviewed the situation regarding collision induced absorption in stellar atmospheres. $b-f$ transitions in small molecules take place mainly in the UV region and will therefore not affect the stellar structure markedly, but can contribute substantially to the UV spectrum. The near infrared collision induced absorption is expected to be of importance for the structure (as well as for the IR spectra) of cool, lowmetallicity dwarfs.

Borysow A. 1994, in: Molecules in the Stellar Environment, U.G. Jorgensen (ed.), Springer, p.209

Brown L.R., Farmer C.B., Rinsland C.P., Toth R.A. 1987, Appl. Optics, 26, 5154

Davis S.P. 1987, Publ. Astron. Soc. Pac., 99, 1105

Davis S.P. 1994, in: Molecules in the Stellar Environment, U.G. Jorgensen (ed.), Springer, p. 397

Husson N., Bonnet B., Scott N.A., Chedin A. 1991, Internal Note, Laboratoire de Meteorologie Dynamique. No. 163

Jorgensen U.G. 1995, in: Astrophysical Applications of Powerful New Atomic Databases, S. Adelman, W. Wiese (eds.), ASP Conference Series, in press

Jorgensen U.G., Jensen P. 1993, J. Mol. Spectrosc., 161, 219

Jorgensen U.G., Larsson M. 1990, A\&A, 238, 424

Kurucz R.L. 1994, in: Molecules in the Stellar Environment, U.G. Jorgensen (ed.), Springer, p. 282

Kurucz R.L., Peytremann E. 1975, SAO Spec. Rept., 362

Kuznetsova L.A., Pazyuk E.A., Stolyarov A.V. 1993, Russian J. Phys. Chem., 67, 2046

Langhoff S.R., Bauschlicher C.W. 1993, Chem. Phys. Lett., 211, 305

Littleton J.E., Davis S.P. 1985, ApJ, 296, 152

Pineiro A.L., Tipping R.H., Chackerian C.Jr. 1987a, J. Mol. Spec., 125, 91

Pineiro A.L., Tipping R.H., Chackerian C.Jr. 1987b, J. Mol. Spec., 125, 184

Rothman L.S., et al. 1992, JQSRT, 48, 469

Tsuji T. 1986, ARA\&A, 24,411

van Dishoeck E.F. 1994, in: Molecules in the Stellar Environment, U.G. Jorgensen (ed.), 